\title{
La littérature en langues congolaises nous permet d'exister souverains
}

Entretien mené par Julien Jeusette

Bienvenu Sene Mongaba

\section{CpenEdition}

Journals

Édition électronique

URL : https://journals.openedition.org/coma/5883

DOI : $10.4000 /$ coma.5883

ISSN : 2275-1742

Éditeur

Institut des textes \& manuscrits modernes (ITEM)

Référence électronique

Bienvenu Sene Mongaba, "La littérature en langues congolaises nous permet d'exister souverains », Continents manuscrits [En ligne], 15 | 2020, mis en ligne le 15 octobre 2020, consulté le 13 janvier 2023. URL : http://journals.openedition.org/coma/5883 ; DOI : https://doi.org/10.4000/coma.5883

Ce document a été généré automatiquement le 13 janvier 2023.

\section{(c) (†) $\odot$}

Creative Commons - Attribution - Pas d'Utilisation Commerciale - Pas de Modification 4.0 International - CC BY-NC-ND 4.0

https://creativecommons.org/licenses/by-nc-nd/4.0/ 


\title{
La littérature en langues congolaises nous permet d'exister souverains
}

\author{
Entretien mené par Julien Jeusette
}

Bienvenu Sene Mongaba

\section{NOTE DE L'ÉDITEUR}

Né en 1967 à Kinshasa, Bienvenu Sene Mongaba est chimiste de formation. Après avoir travaillé en tant que chercheur en biotechnologie, puis en tant qu'enseignant en école secondaire en Belgique, il a créé en 2005 l'ASBL Mabiki à Wavre (Belgique). Cette association a pour but, notamment, de promouvoir les littératures en langues africaines et de travailler à l'amélioration de l'enseignement au Congo. Mabiki n'est pas seulement un centre de formation, c'est également une maison d'édition qui publie des textes littéraires, des ouvrages destinés à l'enseignement et à l'apprentissage des langues africaines. Bienvenu Sene Mongaba a en effet pour objectif la promotion du lingala. Il insiste sur la nécessité d'approfondir l'étude grammaticale et linguistique de cette langue, et y contribue lui-même : en 2013, il soutient une thèse de doctorat en linguistique à l'Université de Gand intitulée « Le Lingala dans l'enseignement des sciences dans les écoles de Kinshasa ». Cette thèse est entièrement rédigée en lingala avec une traduction en français. Il enseigne le lingala, ainsi que la traduction vers cette langue à l'Université Pédagogique Nationale (Congo). Il a enseigné le lingala à l'Université de Gand comme professeur visiteur. Écrivain, il a publié plusieurs romans en lingala aux éditions Mabiki Fwa-Ku-Mputu (Mourir en Europe), Bokobandela, Sanza nguma et Basalela babwaka. Il est aussi auteur de manuels scolaires de chimie et de mathématique en lingala. Il a contribué à la traduction du lingala vers l'anglais du roman Ebamba de Richard Ali A Mutu. 


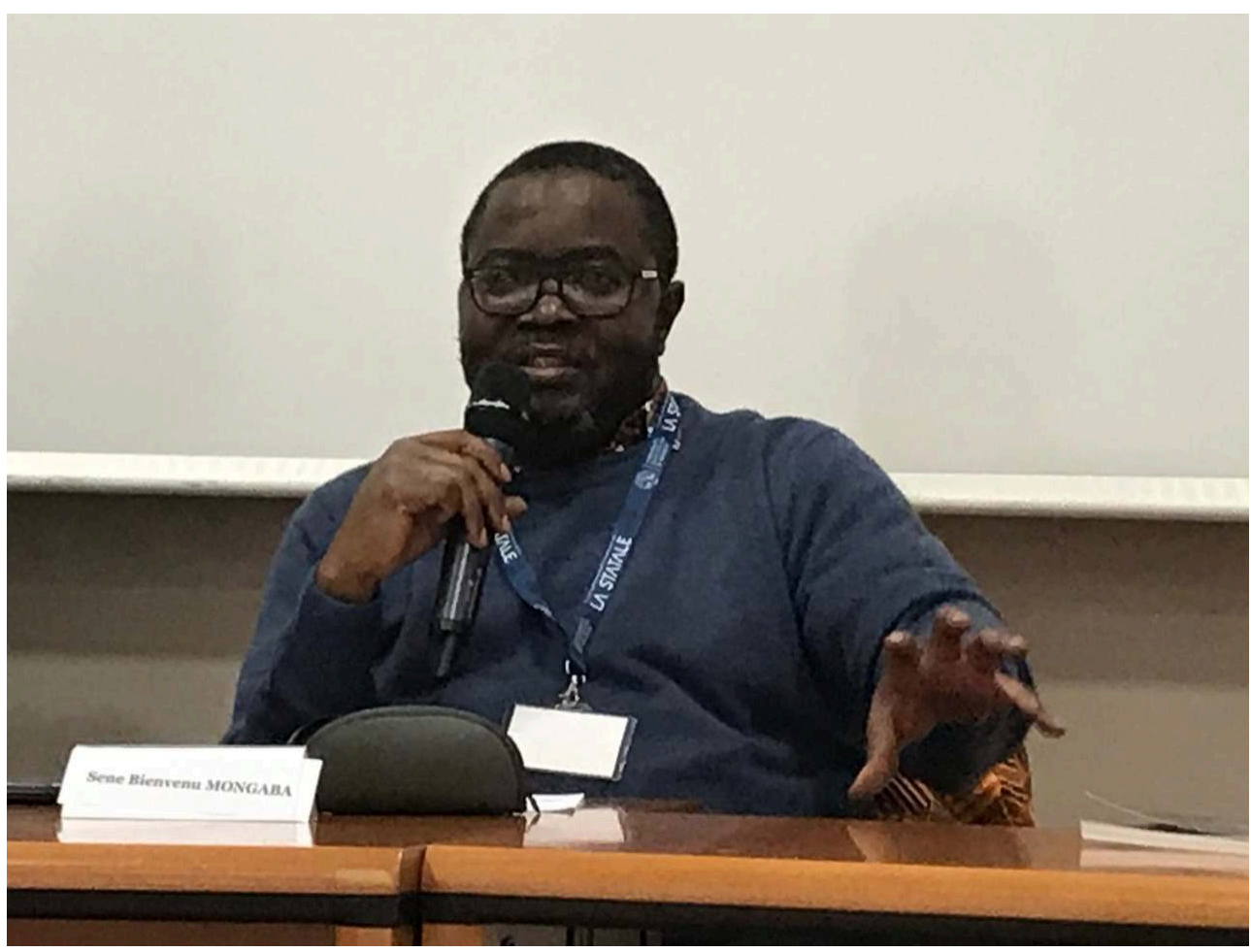

Photo de Silvia Riva

JULIEN JEUSETte : Par le biais de votre association Mabiki, vous êtes depuis longtemps un ardent défenseur des langues africaines et du multilinguisme au Congo. Est-ce que, selon vous, cela a un sens de parler de «littérature congolaise » aujourd'hui, ou faudrait-il plutôt parler de littératures au pluriel (en lingala, en swahili, en kikongo, en français...) ?

Bienvenu Sene Mongaba : L'adjectif «congolaise » qui qualifie cette « littérature » contient déjà les germes de sa propre ambiguïté. Que signifie «littérature congolaise »? Ce terme composé évoque plusieurs acceptions. Il pourra s'agir d'une littérature faite par des Congolais, d'une littérature faite en langues congolaises, d'une littérature destinée aux Congolais d'abord, d'une littérature faite au Congo, d'une littérature faite sur le Congo...

La question de la territorialité et de la langue se pose souvent dans le cadre des pays africains à cause de la langue utilisée pour produire de la littérature dans ces pays. Cependant, de façon générale, l'adjectif qualificatif (chinoise, américaine, bulgare, russe...) se réfère aux langues du pays. En outre, avant d'y répondre, il est opportun de se poser une autre question : «À qui est destinée une production littéraire ? En principe, un auteur s'adresse aux gens de sa société. C'est le niveau local. Comme l'humain est le même partout, les lecteurs des sociétés étrangères à celle de l'auteur pourront accéder à son œuvre à travers la traduction ou l'apprentissage de la langue de l'auteur. Un texte bien écrit atteint l'universel. Partant de l'essence même d'un texte littéraire (raconter une histoire, un élan du cœur...), son parcours normal est de partir du local vers l'universel et non l'inverse.

Plusieurs chercheurs intègrent dans la littérature congolaise des textes écrits en langue française par des Congolais. Pendant longtemps, la tendance était même de considérer ce corpus comme étant «la littérature congolaise ». En revanche, nous 
observons qu'une grande majorité des auteurs congolais qui écrivent en français s'autoexcluent eux-mêmes de la littérature congolaise puisqu'ils ont le regard tourné vers Paris et le lecteur congolais du Congo ne fait pas partie de leur cible première. Le texte s'en ressent quand l'auteur est appelé à expliquer certains faits culturels qu'il n'aurait pas eu à expliquer si son lecteur cible était le Congolais.

À mon avis, la littérature congolaise est donc la littérature écrite dans une langue congolaise à destination première des Congolais. De ce fait, je prends la liberté d'exclure tout ce qui est écrit en français, en anglais ou dans toute autre langue non congolaise, puisqu'à mon avis, le français n'est pas une langue congolaise, même s'il est imposé comme langue officielle et comme langue de l'enseignement. Mais qui suis-je pour le décréter? La seule façon d'imposer cette définition est celle de me cacher derrière l'expression «à mon avis ". Les textes écrits ou dits en kiswahili, en lingala, en kikongo, en tshiluba ou dans une autre langue du Congo forment donc la littérature congolaise. Par ailleurs, la littérature kiswahili englobe les textes en kiswahili faite en RDC, Tanzanie, Kenya, Uganda... alors que la littérature kikongo englobe ceux produits en kikongo dans les deux Congo et en Angola. Cette situation n'est pas propre à l'Afrique, on la retrouve partout dans le monde, puisque la territorialité des citoyens ne correspond pas à la territorialité des langues. Dans cette optique, les textes en français produits par des Congolais font partie de la littérature de langue française (les Français préfèrent leur réserver l'adjectif « francophone »).

J. J. : Pour rester sur la question du multilinguisme, vous faites remarquer dans vos ouvrages et dans vos entretiens que la colonisation passe par l'imposition d'une langue considérez-vous que le Congo, par exemple, devrait aujourd'hui commencer à « oublier » le français ? Et considérez-vous que les langues parlées au Congo sont sur un pied d'égalité, ou est-ce que l'une d'entre elles pourrait jouer un plus grand rôle?

B. S. M.: L'usage du français est contre-productif à plusieurs égards. Il n'est un secret pour quiconque qu'une personne apprend mieux dans sa langue de tous les jours. Au Congo, un enfant parle une langue congolaise dans son quotidien. Une fois qu'il franchit la porte de l'école, on lui interdit à coup de sanctions disciplinaires de parler sa propre langue. L'école indique à l'enfant que sa langue, et donc sa culture n'a pas de valeur. Si l'enfant veut accéder au savoir, il doit tourner son regard vers l'extérieur. L'école congolaise forme l'enfant à ne pas aimer le Congo. Même les savoirs endogènes, héritage millénaire laissé par nos ancêtres, ne sont pas enseignés à l'enfant. Les résultats sont catastrophiques et décriés par tout le monde.

L'école congolaise doit donc enseigner dans les langues congolaises pour deux raisons majeures : permettre à l'apprenant congolais de mieux comprendre ce qui lui est enseigné et d'avoir accès aux savoirs endogènes utiles pour qu'il soit agent du progrès.

Les langues parlées au Congo devraient être considérées sur un pied d'égalité. Je ne vois pas pourquoi un enfant qui est à Mbuji-Mayi où la langue première est le tshiluba devrait par exemple apprendre en lingala. En revanche, pour édifier l'unité nationale, il est judicieux que les trois autres langues nationales soient enseignées comme discipline. Ceci permettra d'avoir des personnes capables de s'exprimer dans les quatre langues nationales. Cela dit, une langue communautaire doit être enseignée sur le territoire de ladite communauté. Il ne s'agit donc pas de choisir une langue pour lui donner un rôle mais de fonder notre démarche sur les réalités du terrain de façon que l'usage de nos langues soit optimal. La société congolaise est 
traditionnellement multilingue. Il nous faut nous appuyer sur les stratégies multilingues qui ont toujours fonctionné au Congo pour pouvoir bâtir une société plus prospère.

J. J. : Votre travail souligne bien que le choix d'une langue est inséparable d'une politique et a des effets majeurs sur l'ensemble des locuteurs - par exemple, enseigner les sciences en français à un public dont ce n'est pas la langue maternelle a des effets délétères. Depuis que vous avez commencé à enseigner le lingala, est-ce que vous constatez des changements dans le rapport de la population aux langues africaines? Est-ce que le gouvernement, les écoles ou les parents qui y envoient leurs enfants sont favorables à un enseignement dans ces langues?

B. S. M. : Je constate énormément de changements. Au début, les parents étaient réticents à ce qu'on enseigne leurs enfants en lingala. Mais au vu des résultats, quand bien même ils continuent à espérer que leurs enfants puissent bien parler le français, ils se rendent compte qu'en adoptant cette approche les enfants acquièrent plusieurs compétences nécessaires et utiles.

L'État ne s'y oppose pas, puisqu'au niveau de la loi, le français et les langues congolaises sont des langues d'enseignement. L'État lui-même a produit plusieurs manuels d'apprentissage des langues congolaises pour les trois premières années du primaire. Malheureusement, je dois reconnaître que l'attitude négative vis-à-vis des langues congolaises de la part des instruits congolais me plonge dans une déception sans nom. Heureusement que nous travaillons dans une optique à long terme et je suis objectivement conscient que l'on finira par revenir aux langues congolaises puisque la structure sociale ne permet pas l'épanouissement dans une langue étrangère.

Il faut avouer que plusieurs instruits congolais sont encore réticents vis-à-vis de l'utilisation de nos langues dans la littérature. Cela tient tout simplement de l'aliénation mentale dont nous sommes victimes de la part de l'école au Congo. À ce jour, l'école, en interdisant aux élèves de s'exprimer en langues congolaises, est la première institution qui se met en travers du chemin de l'éclosion de notre littérature et de notre créativité en général. Il est donc difficile que la littérature écrite en langues congolaises puisse s'étendre et s'épanouir dans la population congolaise. Or c'est l'école qui est le creuset même des lecteurs. C'est à l'école que l'on apprend à lire. En interdisant aux élèves de parler les langues congolaises en son sein, l'école produit des personnes qui n'aiment pas leur propre culture, qui ne connaissent pas leur propre pays et qui n'ont pas d'estime de soi.

Quand nous observons les résultats que ces presque soixante ans d'interdiction de parler lingala ont produits, nous nous posons la question s'il ne faut pas tout simplement fermer l'école. En effet, en RDC, très peu de personnes peuvent prétendre que le français est leur langue première. La quasi-totalité de la population congolaise apprennent le français à l'école et ils sont enseignés dans cette langue. Le Congolais investit douze ans de sa scolarité à n'apprendre que le français et non à mettre à l'épreuve sa capacité d'innover ou de créer.

Le français est certes une langue qui nous permet d'interagir avec quelques pays sur le plan international mais il ne nous permet pas de prendre part à la mondialisation dans le camp de dominants. Ce sont les langues congolaises qui nous tireront de ce guêpier. N'en déplaise aux responsables des écoles. Et la littérature n'y échappera pas. 
Il est plus que temps que l'État congolais oblige les écoles à s'abstenir d'interdire aux enfants de parler les langues congolaises. À défaut, il faudrait même envisager le paradoxe de devoir fermer l'école et inventer une autre institution capable de former des citoyens.

J. J. : La notion d'espace littéraire «mondial » a-t-elle un sens pour vous ? Considérez-vous que les textes littéraires que vous publiez en font (ou en feront) partie, ou est-ce que vous visez un public plus restreint lorsque vous décidez de publier un auteur?

B. S. M. : Oui. Les textes littéraires en langues congolaises que nous publions chez Mabiki ou chez d'autres éditeurs congolais font partie de l'espace littéraire «mondial ». Pour faire partie de l'espace littéraire mondial, nous n'avons pas besoin de demander de permission à qui que ce soit. D'ailleurs personne, nulle part, a demandé d'autorisation pour faire partie de la littérature mondiale.

Je parle toujours du fait que l'on ne peut atteindre l'universel qu'en partant du local. Écrire en lingala pour un public qui parle le lingala ne signifie pas que la portée du livre soit restreinte. Même quelqu'un qui écrit en anglais n'écrit pas pour un milliard de personnes, alors que la population mondiale est déjà à 7 milliards. L'universel est plutôt lié à l'art de raconter et au contenu de l'œuvre littéraire qu'à la langue utilisée.

Ce reproche est souvent avancé par des instruits congolais mais sans fondement. Nous écrivons en français et pourtant nous ne sommes pas lus partout dans le monde. La littérature congolaise ne s'est pas encore imposée dans le monde pour la simple raison qu'elle ne s'est pas encore imposée au Congo. Le fait d'écrire en lingala ne nous empêche pas d'être lus dans le monde. La traduction existe. D'ailleurs, le roman Ebamba de Richard Ali, écrit en lingala, a été traduit en anglais et publié par un éditeur de Californie aux USA. Ce qui compte, c'est la qualité littéraire de l'œuvre et non la langue dans laquelle elle est écrite. Ensuite, ce sont le professionnalisme et les capacités financières consacrés dans la communication et la diffusion de l'œuvre qui déterminent ses chances à l'international.

Innoss'B chante Yo Pe en lingala; il a des millions de vues sur Youtube. La musique congolaise est une leçon pour la littérature congolaise. Il ne faut pas perdre de vue que la littérature en langues congolaises nous permet d'exister souverains n'attendant la reconnaissance que du lecteur congolais. Ce n'est que quand nous aurons de la reconnaissance au Congo que l'international sera prêt à s'intéresser à notre littérature. Je parle des éditeurs et des lecteurs à l'étranger et non des chercheurs universitaires. C'est du local que nous atteignons l'universel et pas l'inverse.

Donc pour répondre à la question, je dirai que nous faisons partie de l'espace littéraire mondial puisque le Congo fait partie du monde.

J. J. : Comment envisagez-vous l'avenir des littératures africaines ?

B. S. M. : En général, les auteurs africains écrivent non seulement en langues européennes mais ont pour cible le lecteur occidental. L'avenir de la littérature en Afrique dépend de la manière dont les acteurs de la littérature écrite et orale intégreront dans leur stratégie le lecteur africain en Afrique. Aussi longtemps qu'ils vont courir derrière une reconnaissance mondiale oubliant que le monde commence dans leur propre rue, cette littérature ne sera que l'affaire des experts. Les auteurs doivent s'adresser aux Africains avec les codes littéraires africains. Aujourd'hui, la chanson congolaise constitue la grande littérature congolaise parce que les auteurs 
comme Lutumba, Koffi Olomide, Reddy Amisi ont produit des œuvres littéraires de haute facture qui mériteraient d'être enseignées dans des écoles.

Il faut reconnaître cependant que la production de livres en langues congolaises est encore très faible. Il est difficile de dresser une liste exhaustive de publications littéraires. Ceci est dû au fait que le circuit de distribution et de diffusion du livre congolais se trouve encore au niveau artisanal. Le réseau de diffusion est faible. Ceci n'est pas propre aux livres écrits en lingala mais c'est la situation générale de l'édition congolaise.

Quoi qu'il en soit, aujourd'hui, la littérature en langues congolaises est en train de se fixer un contour moderne. De plus en plus d'auteurs se frottent à l'exercice de l'écriture dans ces langues. Nous pensons que, dans quelques années, on va assister à des productions de plus en plus conséquentes, non seulement dans le domaine littéraire mais aussi scolaire et scientifique. La question de l'orthographe et du registre trouvera son dénouement dans l'évolution et la réception des publications littéraires et scolaires. Le développement de la littérature en langues congolaises contribuera pour beaucoup dans la renaissance socio-économique du Congo.

Quelques publications aux éditions Mabiki 

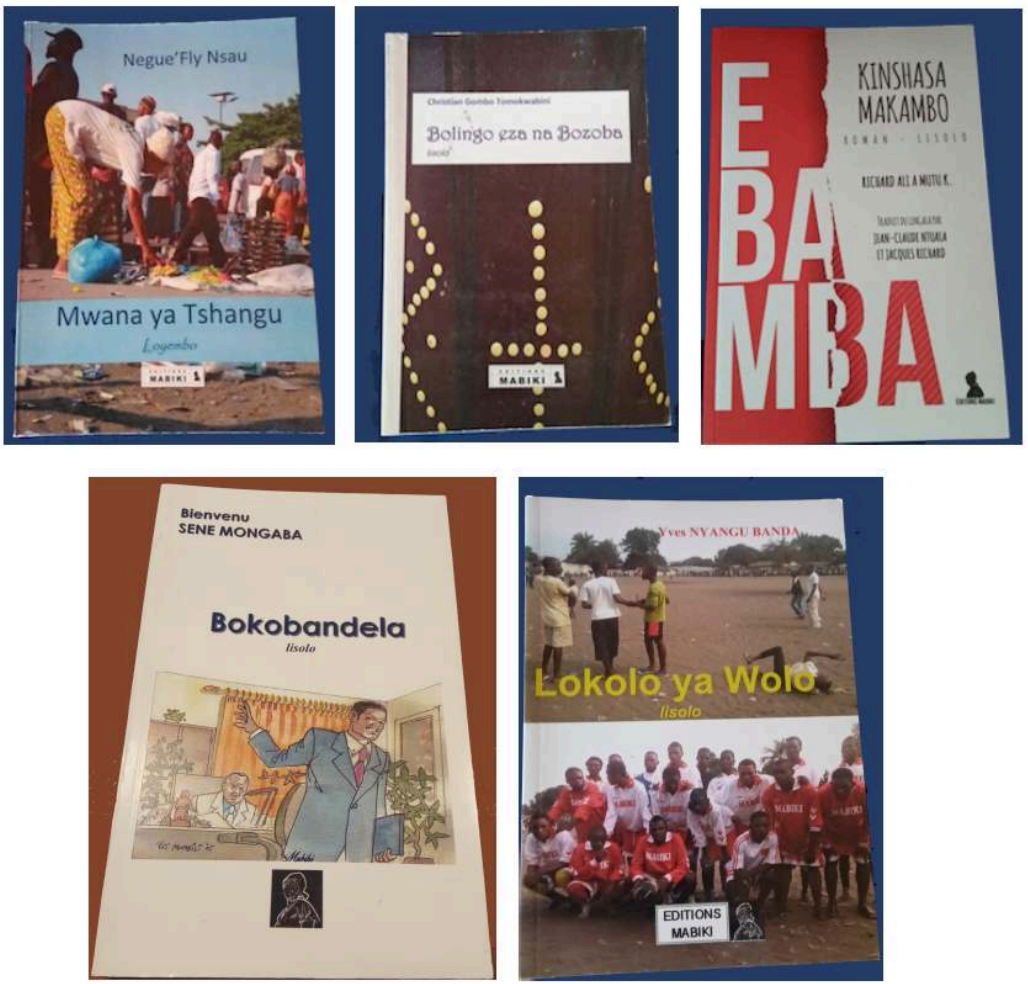

INDEX

Mots-clés : littérature en langues congolaises, multilinguisme congolais, lingala, territorialité de la langue, enseignement des langues, espace littéraire « mondial »

\section{AUTEUR}

\section{BIENVENU SENE MONGABA}

Responsable de l'association ASBL Mabiki (centre de formation et maison d'édition) et professeur de lingala 